\title{
Block Equalization for Single-Carrier Satellite Communications with High-Mobility Receivers
}

\author{
L. Rugini, P. Banelli \\ Dept. of Elect. and Inform. Eng. (D.I.E.I.) \\ University of Perugia \\ Perugia, Italy \\ \{luca.rugini, paolo.banelli\}@diei.unipg.it
}

\author{
M. Berioli \\ Institute of Communications and Navigation \\ German Aerospace Center (DLR) \\ Wessling, Germany \\ matteo.berioli@dlr.de
}

\begin{abstract}
We compare some block equalizers for singlecarrier satellite systems subject to hard propagation conditions such as high Doppler spread, non-line-of-sight (NLOS), and nonnegligible multipath fading. These impairments are relevant when the very high speed of a mobile receiver forces to use a nondirectional antenna. First, we compare low-complexity timedomain and frequency-domain block equalizers. Then, we propose a novel frequency-domain equalizer based on a fast Fourier transform (FFT) with size higher than the length of the processed block. Thanks to the increased frequency resolution, the proposed equalizer yields improved performance with respect to the conventional frequency-domain equalizer. Simulation results validate the effectiveness of the proposed technique.
\end{abstract}

Keywords-Frequency-domain equalization; Doppler spread; NLOS; multipath; satellite communications.

\section{INTRODUCTION}

Satellite transmissions to mobile receivers are becoming more and more common, not only in the frequency bands commonly used in the past (S- and L-band), but also in the Ku-band (10-12 GHz), where a direct line-of-sight (LOS) to the satellite is normally required. In this context, directive antennas are usually adopted for Ku-band communications. Anyway, mobile and portable receivers cannot carry bulky highly-directional antennas, such as dish or phased array antennas. In this view, many researchers and companies are intensively working to develop smaller and flat antennas for satellite receivers, such as cars, buses, and trains, in $\mathrm{Ku}$ and Ka bands.

However, the reduced size of mobile receivers forces the antenna gain patterns to have a wide main beam (up to $30^{\circ}$ $40^{\circ}$ ) and non-negligible side beams, with a consequent reception of multipath signals produced by reflection and scattering due to surrounding objects. In addition, in case of receivers with high mobility, it is easier to steer a low directive antenna in the satellite direction, since its wide main beam can tolerate a lower precision in the pointing direction. On the other hand, deliberate mispointing failures (e.g., in many cases the elevation angle is kept fixed) may also introduce additional multipath, i.e., non-LOS (NLOS) reception. Multipath propagation may be experienced also in case of unobstructed LOS reception, due to high levels of humidity in the atmosphere, e.g., in case of clouds, rain or snow. For instance,

This work was supported by the European Union under the project "Satellite Communications Network of Excellence," phase 2 (SatNEx-2). the effect of rain is shown in [1] for a Ka-band terrestrial link. As a consequence of these multiple propagation paths, the receiver can benefit from channel equalization, even for single-carrier satellite signal reception at high operational frequencies, like the Ku-band.

In this paper, we consider equalization techniques that are capable to handle both multipath propagation due to nondirective antennas and Doppler spread due to receiver motion. As a reference scenario, we consider the Digital Video Broadcasting - Satellite (DVB-S), and more specifically the DVB-S - Second Generation (DVB-S2) standard [2]. Although the DVB-S set of standards, DVB-S, DVB-S2, and DVB-RCS (return channel via satellite), working in the $\mathrm{Ku}$ and $\mathrm{Ka}$ bands, was not initially meant for mobile receivers and transmitters, work is in progress in adapting these standards to mobile communications [3]. For example, DVB-RCS is currently under revision in the DVB Forum to enable its operation in mobile terminals. In addition, in February 2007, the DVB steering board approved some specifications of a new standard, called DVB-SH (Satellite services to Handheld), to enable satellite broadcasting services to portable and handheld devices. However, we warn that the chosen scenario is not crucial to the effectiveness of the proposed equalization approaches, which could potentially be used in any other single-carrier satellite system for handheld scenarios.

We herein consider block linear minimum mean-squared error (LMMSE) equalizers that exploit soft interference cancellation to remove the multipath effect of the previous equalized block. We show that, under a computational complexity constraint, frequency-domain (FD) equalizers generally outperform time-domain (TD) equalizers. We also propose a novel FD-like equalizer based on a fast Fourier transform (FFT) with size higher than the length of the processed block. Simulation results illustrate that the proposed equalizer leads to improved performance with respect to conventional equalizers.

\section{System ModeL}

We briefly summarize the physical layer of the DVB-S2 standard [2]. The data are parsed in frames, each one of length $D=1440$ symbols. We denote with $\mathbf{d}$ the $D \times 1$ vector that contains the data symbols of a generic frame. In [2], two transmission configurations are possible, either with pilots or without pilots. Since we are dealing with time-varying channels, we assume that pilots are transmitted, so that the receiver can update its channel estimate. Hence, we assume 
that a known pilot block $\mathbf{p}$, of length $P=36$ symbols, is periodically inserted between two consecutive frames [2]. We also assume QPSK, i.e., each element of $\mathbf{d}$ is drawn from a $\{( \pm 1 \pm j) / \sqrt{2}\}$ constellation, with covariance $\mathbf{C}_{\mathbf{d d}}=\mathbf{I}_{D}$.

The signal stream is transmitted through a time-varying multipath channel $h_{c}(t, \tau)$, whose discrete-time equivalent impulse response is

$$
h[n, l]=h_{c}\left(n T_{\mathrm{S}}, l T_{\mathrm{S}}\right),
$$

where $T_{\mathrm{S}}$ is the sampling period. Throughout the paper, we assume that the channel amplitudes are complex Gaussian distributed with either a zero or a non-zero mean, i.e., either NLOS or LOS propagation, giving rise to Rayleigh or Rice fading, respectively. We also assume that the maximum delay spread $L$ is smaller than or equal to the pilot duration $P$. This implies $h[n, l]=0$ when $l>P$.

By assuming time and frequency synchronization at the receiver side, the input-output relation can be expressed by

$$
\overline{\mathbf{y}}=\overline{\mathbf{H}} \overline{\mathbf{x}}+\overline{\mathbf{i}}+\overline{\mathbf{n}},
$$

where $\overline{\mathbf{y}}$ is the received block of size $D+P, \overline{\mathbf{H}}$ is a lower triangular square matrix of dimension $D+P$ that represents the channel, with element in position $(n, l)$ defined by

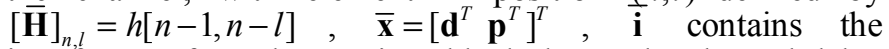
interference from the previous block due to the channel delay spread, and $\overline{\mathbf{n}}$ is the additive white Gaussian noise (AWGN) vector with covariance $\mathbf{C}_{\overline{\mathbf{n n}}}=E\left\{\overline{\mathbf{n}} \overline{\mathbf{n}}^{H}\right\}=\sigma_{n}^{2} \mathbf{I}_{D+P}$. In this paper, we assume that the channel $h[n, l]$ is perfectly known to the receiver. In practice, $h[n, l]$ should be estimated, for example, by exploiting the known pilot symbols [4]. We also assume that the interference from the previous block is known and perfectly compensated_for. This assumption is realistic because $L \leq P$ : hence $\overline{\mathbf{i}}$ depends only on the pilot block $\mathbf{p}$, which is known, and on the channel $h[n, l]$, which is assumed known. As a consequence, $\overline{\mathbf{i}}$ can be reconstructed and subtracted. For our scopes, we can simply assume that $\overline{\mathbf{i}}=\mathbf{0}$ in (2). We do not consider the interference induced by channel estimation errors, which will be subject of future investigation.

Channel equalizers can be grouped into two broad categories: serial equalizers and block equalizers. The difference between these two classes is that serial equalizers estimate each transmitted symbol individually, whereas block equalizers jointly estimate a group of consecutive transmitted symbols. In this paper, we consider block equalization methods. Therefore, some of the data contained into the unknown vector $\mathbf{d}$ are estimated jointly. Since the dimension $D$ of $\mathbf{d}$ is quite high, to reduce the memory requirements, we design block equalizers that estimate a subblock of $\mathbf{d}$ with smaller dimension. Specifically, the transmitted block $\mathbf{d}$ is parsed into $M$ equal-length subvectors $\{\mathbf{d}[m]\}$, each one with size $N=D / M$. The $n$th element of $\mathbf{d}[m]$ is defined as

$$
[\mathbf{d}[m]]_{n}=[\mathbf{d}]_{(m-1) N+n}, \quad n=1, \ldots, N, \quad m=1, \ldots, M .
$$

Due to the finite support of the time-varying channel impulse response in (1), which translates into a banded matrix $\mathbf{H}$ in (2), only a limited number of elements of the received vector $\overline{\mathbf{y}}$ contain information about a specific subblock $\mathbf{d}[m]$. We can collect these useful elements in an $(N+P)$-dimensional vector $\mathbf{y}[m]$ expressed by

$$
[\mathbf{y}[m]]_{n}=[\overline{\mathbf{y}}]_{(m-1) N+n}, \quad n=1, \ldots, N+P, \quad m=1, \ldots, M,
$$

thereby obtaining from (2) a new input-output relation expressed by

$$
\mathbf{y}[m]=\mathbf{H}[m] \mathbf{x}[m]+\mathbf{i}[m]+\mathbf{n}[m],
$$

where

$$
\mathbf{H}[m]=[\overline{\mathbf{H}}]_{(m-1) N+1: m N+P,(m-1) N+1: m N+P}
$$

is a square matrix with dimension $N+P$, where $[\mathbf{A}]_{a: b, c: d}$ denotes the submatrix of $\mathbf{A}$ obtained by selecting the rows from $a$ to $b$ and the columns from $c$ to $d$,

$$
\mathbf{x}[m]=\left[\mathbf{d}[m]^{T} \mathbf{d}_{\text {first }}[m+1]^{T}\right]^{T}
$$

is a vector of length $N+P$, where $\mathbf{d}_{\text {first }}[m+1]=[\mathbf{d}[m+1]]_{1: P}$, represents the first $P$ samples that belong to the "future" data subblock, i.e., that with index $m+1$,

$$
\mathbf{i}[1]=\mathbf{0}, \quad \mathbf{i}[m]=\mathbf{H}_{\text {last }}[m] \mathbf{d}_{\text {last }}[m-1], \quad m=2, \ldots, M,
$$

where $\mathbf{d}_{\text {last }}[m-1]=[\mathbf{d}[m-1]]_{N-P+1: N}$, represents the last $P$ samples of the "past" data subblock, with index $m-1$,

$$
\mathbf{H}_{\text {last }}[m]=[\overline{\mathbf{H}}]_{(m-1) N+1: m N+P,(m-1) N-P+1:(m-1) N},
$$

is the $(N+P) \times P$ matrix that models the channel seen by the past data, and

$$
[\mathbf{n}[m]]_{n}=[\overline{\mathbf{n}}]_{(m-1) N+n}, \quad n=1, \ldots, N+P, \quad m=1, \ldots, M,
$$

is the AWGN contribution on the considered block.

We observe that in (5), similarly to (2) we also have an interference term $\mathbf{i}[m]$, expressed by (8). However, differently from (2), in this case the interference depends on the previous data subvector $\mathbf{d}[m-1]$, which is unknown. As a consequence, this type of interference, called interblock interference (IBI), cannot be neglected. Equations (5) and (7) form the basis that allows to estimate $\mathbf{d}[\mathrm{m}]$ from $\mathbf{y}[\mathrm{m}]$.

\section{BLOCK EQUALIZATION}

The block equalizers we consider provide an estimate of d $[m]$ sequentially, starting from $m=1$ up to $m=M$. To estimate the generic $m$ th block $\mathbf{d}[m]$, we firstly construct a soft estimate of the IBI term from the previously detected block. By denoting with $\hat{\mathbf{d}}[m-1]$ the soft estimate of $\mathbf{d}[m-1]$ at the output of the $(m-1)$ th equalizer, we reconstruct the soft data as

$$
\tilde{\mathbf{d}}[m-1]=\frac{\tanh (C \operatorname{Re}(\hat{\mathbf{d}}[m-1]))+j \tanh (C \operatorname{Im}(\hat{\mathbf{d}}[m-1]))}{\sqrt{2}},
$$

where $C$ is a positive constant. We use the hyperbolic tangent because it allows to tune the soft estimation, which tends to hard estimation for $C \rightarrow+\infty$. By using the last part of $\tilde{\mathbf{d}}[m-1]$, the reconstructed IBI term is obtained by

$$
\tilde{\mathbf{i}}[m]=\mathbf{H}_{\text {last }}[m] \tilde{\mathbf{d}}_{\text {last }}[m-1]
$$

since $\mathbf{H}_{\text {last }}[m]$ is assumed known. The IBI term is then subtracted from the received signal $\mathbf{y}[\mathrm{m}]$ in $(5)$, which gives

$$
\mathbf{z}[m]=\mathbf{y}[m]-\tilde{\mathbf{i}}[m] \approx \mathbf{H}[m] \mathbf{x}[m]+\mathbf{n}[m] .
$$

The model of (13) can now be used to design TD and FD LMMSE equalizers.

\section{A. Time-Domain LMMSE Equalization}

From (7), it is clear that an estimate of $\mathbf{d}[\mathrm{m}]$ can be extracted from the corresponding estimate of $\mathbf{x}[\mathrm{m}]$. By performing the equalization in the TD, the LMMSE estimate of $\mathbf{x}[m]$ is expressed by [5]

$$
\hat{\mathbf{x}}[m]=\mathbf{H}[m]^{H}\left(\mathbf{H}[m] \mathbf{H}[m]^{H}+\sigma_{n}^{2} \mathbf{I}_{N+P}\right)^{-1} \mathbf{z}[m] .
$$

A direct implementation of (14) is impractical, since it would require $O\left(M(N+P)^{3}\right)$ complex operations to perform the $M$ matrix inversions. Although we can increase the block parsing factor $M$ to reduce the value of $N$, we cannot reduce the 
value of $P$, which can be interpreted as the maximum length of the multipath channel. As a result, the complexity of the direct implementation of (14) is at least cubic in $P$.

The most convenient way to obtain $\hat{\mathbf{x}}[\mathrm{m}]$ is to exploit the band triangular structure of $\mathbf{H}[\mathrm{m}]$ given by the finite length of the multipath channel. In the following, we briefly describe this procedure. By defining

$$
\mathbf{C}[m]=\mathbf{H}[m] \mathbf{H}[m]^{H}+\sigma_{n}^{2} \mathbf{I}_{N+P},
$$

the equalizer can firstly solve for $\gamma[m]$ the linear system expressed by

$$
\mathbf{C}[m] \gamma[m]=\mathbf{z}[m],
$$

and then obtain $\hat{\mathbf{x}}[m]$ as

$$
\hat{\mathbf{x}}[m]=\mathbf{H}[m]^{H} \boldsymbol{\gamma}[m] .
$$

The procedure expressed by (15)-(17) is convenient as long as the linear system in (16) can be solved with low computational cost. However, by definition, $\mathbf{H}[\mathrm{m}]$ is banded lower triangular [6] with lower bandwidth equal to $L$, and hence also the matrix $\mathbf{C}[m]$ in $(15)$ is banded. Since both the upper and the lower bandwidth are equal to $L$, the bandwidth of $\mathbf{C}[m]$ is equal to $2 L+1$. Hence, by exploiting a band factorization of $\mathbf{C}[m]$ (see, e.g., [7]), we can solve the $M$ linear systems of (16) in $O\left(M(N+P) L^{2}\right)$ operations, which is only linear in $N+P$. This represents a significant improvement for short multipath channels, where $L<<P$. However, for long channels, the TD LMMSE equalizer is still rather complex, since its complexity is at least cubic in $L$.

A complexity reduction is possible by considering, instead of the true channel matrix $\mathbf{H}[\mathrm{m}]$, an approximated channel matrix $\hat{\mathbf{H}}[\mathrm{m}]$ that neglects some of the paths of the multipath channel. As an example, we can approximate the TD multipath channel by considering only its first paths, which are usually the strongest ones, at least for exponentiallydecaying power-delay profiles. If we denote by $R$ the number of retained paths in $\mathbf{H}[\mathrm{m}]$, we can reduce the complexity up to $O\left(M(N+P) R^{2}\right)$. The price paid for this complexity reduction is a performance loss due to the channel modeling error $\mathbf{E}[m]=\hat{\mathbf{H}}[m]-\mathbf{H}[m]$ introduced by multipath truncation. Intuitively, this error is small when the neglected paths have small energy, as it happens for channels with decaying powerdelay profiles. However, the performance loss also depends on the number of neglected paths, since each path contributes individually to the frequency-selectivity of the channel. In Section IV, we will use simulation results to quantify the BER penalty caused by this channel mismatch.

\section{B. Frequency-Domain LMMSE Equalization}

An alternative is to design the LMMSE equalizer in the FD. By denoting with $\mathbf{F}_{N}$ the unitary FFT matrix of size $N$, we define the vectors $\mathbf{z}_{\mathrm{f}}[m]=\mathbf{F}_{N+P} \mathbf{z}[m], \mathbf{x}_{\mathrm{f}}[m]=\mathbf{F}_{N+P} \mathbf{x}[m]$, $\mathbf{n}_{\mathrm{f}}[m]=\mathbf{F}_{N+P} \mathbf{n}[m]$, and the matrix

$$
\mathbf{H}_{\mathrm{f}}[m]=\mathbf{F}_{N+P} \mathbf{H}[m] \mathbf{F}_{N+P}^{H} .
$$

From these definitions, it is easy to show that (13) becomes

$$
\mathbf{z}_{\mathrm{f}}[m] \approx \mathbf{H}_{\mathrm{f}}[m] \mathbf{x}_{\mathrm{f}}[m]+\mathbf{n}_{\mathrm{f}}[m],
$$

and hence the same approach used for TD equalization can be used. The main difference is that $\mathbf{H}[\mathrm{m}]$ is banded triangular, whereas $\mathbf{H}_{\mathrm{f}}[\mathrm{m}]$ is a full matrix, which would lead to a cubic complexity in $N+P$. However, the matrix $\mathbf{H}_{\mathrm{f}}[m]$ in (18), which represents the FD effect of the channel, can be well approximated as banded. In the following, we briefly motivate this approximation. In general, time variant channels are characterized by Doppler spectra with a limited frequency support. Due to the limited Doppler support, the $k$ th frequency component of the output signal is mainly affected by only those input signal frequency components that are close to the $k$ th one. Mathematically, $\left[\mathbf{z}_{\mathrm{f}}[\mathrm{m}]\right]_{k}$ in (19) is obtained from the elements $\left\{\left[\mathbf{x}_{\mathrm{f}}[m]\right]_{q}\right\}$ with $q \approx k$. If we assume that only $2 Q+1$ elements are relevant, for $k-Q \leq q \leq k+Q$, we can assume that $\mathbf{H}_{\mathrm{f}}[\mathrm{m}]$ is almost banded, with bandwidth equal to $2 Q+1$. As a result, the approximation of $\mathbf{H}_{\mathrm{f}}[\mathrm{m}]$ with its banded version $\hat{\mathbf{H}}_{\mathrm{f}}[\mathrm{m}]$ is reasonable. This fact has been previously observed in [8] for multicarrier systems. Since the bandwidth of $\hat{\mathbf{H}}_{\mathrm{f}}[m]$ is $2 Q+1$, also

$$
\hat{\mathbf{C}}_{\mathrm{f}}[m]=\hat{\mathbf{H}}_{\mathrm{f}}[m] \hat{\mathbf{H}}_{\mathrm{f}}[m]^{H}+\sigma_{n}^{2} \mathbf{I}_{N+P}
$$

is banded, with bandwidth equal to $4 Q+1$. Similarly to the previously described TD approach, the FD LMMSE equalizer firstly solves the linear system

$$
\hat{\mathbf{C}}_{\mathrm{f}}[m] \hat{\boldsymbol{\gamma}}_{\mathrm{f}}[m]=\hat{\mathbf{z}}_{\mathrm{f}}[m],
$$

and then obtains $\hat{\mathbf{x}}[m]$ as

$$
\hat{\mathbf{x}}[m]=\mathbf{F}_{N+P}^{H} \hat{\mathbf{H}}_{\mathrm{f}}[m]^{H} \hat{\boldsymbol{\gamma}}_{\mathrm{f}}[m] .
$$

Taking into account the cost of the FFT processing, the computational complexity of this FD approach is roughly $O\left(M(N+P)\left(\log (N+P)+Q^{2}\right)\right)$, and consequently depends on the chosen parameter $Q$. Intuitively, the integer parameter $Q$ should be chosen proportionally to the maximum channel Doppler spread $f_{\mathrm{D}}$. However, since $\mathbf{H}_{\mathrm{f}}[\mathrm{m}]$ is not perfectly banded, we are not constrained to select a specific value of $Q$, which can be kept small to reduce the overall complexity. Interestingly, also the extreme choice $Q=0$ is possible, leading to a diagonal linear system in (21).

\section{Interpolated Frequency-Domain LMMSE Equalization}

Both TD and FD equalization of single-carrier systems are quite common approaches, especially for time-invariant channels [4][9]. In this subsection, we propose a new version of the FD LMMSE equalizer that is based on an FFT of size $U$, with $U>N+P$. The motivation of using a higher-size FFT is the increased frequency resolution, which can be exploited in the equalization step. This finer resolution can lead to smaller values of $Q$ to obtain a given performance. Since the complexity of FD equalizers is linear in the FFT size and quadratic in $Q$, the reduction of $Q$ can balance the increased FFT size. We call this specific frequency domain as interpolated frequency domain (IFD). Moreover, we define $\mathbf{z}_{\text {if }}[m]=\mathbf{F}_{U}\left[\mathbf{z}[m]^{T} \mathbf{0}_{U-(N+P)}^{T}\right]^{T}$,

$$
\mathbf{H}_{\mathrm{if}}[m]=\mathbf{F}_{U}\left[\begin{array}{cc}
\mathbf{H}[m] & \mathbf{0} \\
\mathbf{0} & \mathbf{0}
\end{array}\right] \mathbf{F}_{U}^{H},
$$

$\mathbf{x}_{\mathrm{if}}[\mathrm{m}]=\mathbf{F}_{U}\left[\mathbf{x}[\mathrm{m}]^{T} \boldsymbol{\chi}^{T}\right]^{T}$, where $\boldsymbol{\chi}$ is a vector of dimension $U-(N+P)$, and $\mathbf{n}_{\mathrm{if}}[m]=\mathbf{F}_{U}\left[\mathbf{n}[m]^{T} \mathbf{0}_{U-(N+P)}^{T}\right]^{T}$. From (13), we obtain the input-output relation in the IFD, as expressed by

$$
\mathbf{z}_{\text {if }}[m] \approx \mathbf{H}_{\text {if }}[m] \mathbf{x}_{\text {if }}[m]+\mathbf{n}_{\text {if }}[m] .
$$

It is worth noting that, because of the zeros in the right-hand side of (23), the product $\mathbf{H}_{\text {if }}[\mathrm{m}] \mathbf{x}_{\text {if }}[\mathrm{m}]$ in (24) does not depend on $\chi$. Hence, since we will use (24) to design an LMMSE equalizer, we choose $\chi$ as a dummy random vector, independent from $\mathbf{x}[m]$, with covariance $E\left\{\chi \chi^{H}\right\}=\mathbf{I}_{U-(N+P)}$. This choice simplifies the equalizer, because $\mathbf{x}_{\text {if }}[m]$ is white. In addition, also in this case we approximate the channel matrix $\mathbf{H}_{\text {if }}[m]$ by using its banded version $\hat{\mathbf{H}}_{\text {if }}[\mathrm{m}]$ obtained by retaining only the $2 Q+1$ principal diagonals. As a second 
approximation, we consider the IFD noise as white:

$$
\mathbf{C}_{\mathbf{n}_{\mathrm{if}} \mathbf{n}_{\mathrm{if}}}=\mathbf{F}_{U}\left[\begin{array}{cc}
\sigma_{n}^{2} \mathbf{I}_{N+P} & \mathbf{0} \\
\mathbf{0} & \mathbf{0}
\end{array}\right] \mathbf{F}_{U}^{H} \approx \frac{N+P}{U} \sigma_{n}^{2} \mathbf{I}_{U} .
$$

Therefore, we have

$$
\begin{aligned}
\hat{\mathbf{C}}_{\text {if }}[m]= & \hat{\mathbf{H}}_{\text {if }}[m] \hat{\mathbf{H}}_{\text {if }}[m]^{H}+\frac{N+P}{U} \sigma_{n}^{2} \mathbf{I}_{U}, \\
& \hat{\mathbf{C}}_{\text {if }}[m] \hat{\gamma}_{\text {if }}[m]=\hat{\mathbf{z}}_{\text {if }}[m],
\end{aligned}
$$

and then we obtain $\hat{\mathbf{x}}[m]$ as the first $N+P$ elements of

$$
\hat{\mathbf{x}}_{\text {if }}[m]=\mathbf{F}_{U}^{H} \hat{\mathbf{H}}_{\text {if }}[m]^{H} \hat{\boldsymbol{\gamma}}_{\text {if }}[m] .
$$

The computational complexity of the IFD equalizer is $O\left(M U\left(\log U+Q^{2}\right)\right)$, which looks higher than that of the FD equalizer. However, we have already underlined that, for a given performance, in the IFD case the parameter $Q$ could be smaller than in the FD. Moreover, to keep the approximation (25) reasonable, $U$ should not be much bigger than $N+P$. Since the complexity is quadratic in $Q$ and linear in the FFT size, which equalizer is less complex depends on the specific choice of the various parameters.

We observe that an FD equalizer based on a higher-size FFT has been independently proposed in [10], which proposes a TD window design that reduces the effects of the Doppler spread. Our main difference with [10] is the different parameterization of the channel matrix $\mathbf{H}_{\mathrm{if}}[\mathrm{m}]$ in (23). In addition, since we do not consider TD windowing, our approach is independent from the channel statistics, i.e., our equalizer does not require the knowledge of the power-delay profile and of the Doppler spectrum of the channel.

It is worth noting that, due to the zero padding of the TD channel matrix in (23), we created an artificial discontinuity of the time variability of the channel. Therefore, we expect that the channel matrix $\mathbf{H}_{\text {if }}[m]$ in (23) is "less banded" than $\mathbf{H}_{\mathrm{f}}[m]$ in (18). However, the increased resolution due to the higher FFT size can potentially balance the increased band approximation error. Therefore, we will resort to simulation results to assess the performance-complexity comparison among the various equalizers.

\section{Simulation Results}

We compare by simulations the BER performance of the different equalizers described in Section III. To be compliant with [2], we set $D=1440, P=36$, the symbol rate $f_{\mathrm{S}}=27.5$ $\mathrm{MHz}$, and the carrier frequency $f_{\mathrm{C}}=12 \mathrm{GHz}$. The data constellation is QPSK. To the best of the authors' knowledge, a complete characterization of the multipath phenomenon for $\mathrm{Ku}$-band communications is still absent. To overcome this kind of problems, usually researchers try to infer the channel parameters from measurements done in adjacent bands. For instance, the authors of [11] exploit channel measurements in the L-band to characterize the channel behavior in Ku-band. Therefore, we follow a similar approach, and exploit the multipath characterization obtained in [12] for the L-band. We assume that the channel has an exponential power-delay profile, with rms delay spread $\sigma_{\tau}=0.145 \mu \mathrm{s}$, truncated to a maximum of $L=12$ channel paths. We also assume a Jakes' Doppler spectrum shape with maximum Doppler spread $f_{\mathrm{D}}=f_{\mathrm{C}} V / c$, where $V$ is the mobile speed and $c=3 \cdot 10^{8}$ $\mathrm{m} / \mathrm{s}$ is the speed of light. When a LOS component is present, we assume that this LOS is time invariant, and that its temporal position coincides with that of the first NLOS component. We denote with $K$ the ratio between the power of the LOS component and the sum of the NLOS components' powers. At the receiver side, the parameter used in (11) for soft cancellation is $C=5$. This produces a big cancellation of the soft data, because $\tanh (C x) \approx C x$ for small $x$. For all the receivers (except for the TD equalizer that uses all the taps), we have inserted a regularization parameter to prevent an excessive amplification of the approximation error at high signal-to-noise ratio (SNR). Therefore, the noise variance used in the equalizer has been chosen ad hoc, using the rule-ofthumb $\hat{\sigma}_{n}^{2}=\max \left\{\sigma_{n}^{2}, 10^{-2}\right\}$.

To compare the complexities of the different equalizers, we define $F$ as the average number of complex operations per symbol. A detailed analysis similar to that in [7] reveals that, neglecting the FFT complexity, $F=\left(2 R^{2}+11 R+4\right)(1+P / N)$ for TD equalizers, $F=\left(4 Q^{2}+22 Q+4\right)(1+P / N)$ for $\mathrm{FD}$ equalizers, and $F=\left(4 Q^{2}+22 Q+4\right) U / N$ for IFD equalizers.

In the first set of simulations, we fix $M=8$, which leads to $N+P=216$. The IFD parameter $U=256$, which is the closest power of two greater than $N+P=216$, has been selected. Fig. 1 illustrates the BER performance of different equalizers when $V=300 \mathrm{Km} / \mathrm{h}$ and $K=0$ (Rayleigh fading). It is clear that TD equalizers highly suffer from the error modeling due to considering only $R<L$ channel paths, leading to an error floor with BER $>10^{-1}$. On the contrary, equalizers designed in the FD are more robust to the modeling error. Specifically, the error floor is at $\mathrm{BER} \approx 2 \cdot 10^{-4}$ for the FD equalizers, and below $\mathrm{BER} \approx 10^{-5}$ for the IFD equalizers. This means that, at high SNR, IFD equalizers are preferable. At low SNR, both FD and IFD equalizers have similar performance. It is also worth noting that an increase of $Q$ produces a relatively small performance gain, compared to the complexity increase. Fig. 2 displays the BER as a function of the mobile speed $V$. For all the detectors, the BER is almost independent of $V$. This means that the considered equalizers can be adopted also when the mobile speed is very high. However, a higher speed increases the time variability of the channel, and consequently the channel estimation errors (not considered in this paper) should be more relevant. Fig. 3 exhibits the BER in Rician channels as a function of the Rice factor $K$. It is worth noting that the performance difference between the FD and the IFD equalizers increases with $K$. This can be explained as follows. In the Rice case, the BER floor of FD equalizers, despite lower than for Rayleigh channels, starts at a lower SNR. This early-SNR floor effect, which is more evident for increasing $K$, partially reduces the FD performance gain given by the Rice factor. On the contrary, IFD equalizers do not experience any early floor.

In the second set of simulations, we compare performance and complexity as a function of the number of subblocks $M$. The IFD parameter $U$ is chosen as the closest power of two greater than $N+P$. Fig. 4 shows that the performance of TD and FD equalizers have a small sensitivity to the choice of the number of subblocks $M$. On the contrary, the IFD equalizers are more sensitive to the choice of $M$. High values of $M$, which are related to a small memory size, tends to degrade the performance. Furthermore, the computational complexity, illustrated in Fig. 5, is almost independent from the subblock size. Indeed, the banded equalizer herein considered have linear complexity in the subblock size, and hence the complexity per symbol is approximately constant with $M$.

\section{CONCLUSIONS}

We have investigated some equalization strategies for single-carrier satellite communications systems in the presence of multipath propagation and significant Doppler spread. Both 
LOS and NLOS scenarios have been considered. IFD equalization seems to be a promising approach to enable handheld satellite communications. These results should be confirmed by further studies, which could benefit from powerdelay profiles obtained from measurements in the Ku band. In addition, the impact of practical channel estimation techniques is currently under investigation.

\section{ACKNOWLEDGMENT}

We would like to thank Prof. Geert Leus and the anonymous reviewers for their useful comments on a previous version of this paper.

\section{REFERENCES}

[1] H. Xu, T. S. Rappaport, R. J. Boyle, and J. H. Schaffner, "Measurements and models for 38-GHz point-to-multipoint radiowave propagation," IEEE J. Select. Areas Commun., vol. 18, pp. 310-321, Mar. 2000.

[2] Digital Video Broadcasting (DVB): Second generation framing structure, channel coding and modulation system for broadcasting, interactive services, news gathering and other broadband satellite applications, ETSI EN 302307 v1.1.1, Mar. 2005.

[3] S. Scalise, Study of DVB-S(2)/DVB-RCS broadband mobile system: Propagation channel models, DLR Technical Note, Apr. 2006.

[4] J. G. Proakis, Digital Communications, 4th ed., McGraw-Hill, New York, 2001.

[5] S. M. Kay, Fundamentals of Statistical Signal Processing: Estimation Theory, Prentice-Hall, Inc., Upper Saddle River, NJ, USA, 1993.

[6] G. H. Golub and C. F. Van Loan, Matrix Computations, 3rd ed., Johns Hopkins Univ. Press, 1996.

[7] L. Rugini, P. Banelli, and G. Leus, "Simple equalization of time-varying channels for OFDM", IEEE Commun. Lett., vol. 9, pp. 619-621, July 2005.

[8] W. G. Jeon, K. H. Chang, and Y. S. Cho, "An equalization technique for orthogonal frequency-division multiplexing systems in time-variant multipath channels," IEEE Trans. Commun., vol. 47, pp. 27-32, Jan. 1999.

[9] H. Sari, G. Karam, and I. Jeanclaude, "Transmission techniques for digital terrestrial TV broadcasting," IEEE Commun. Mag., vol. 33, pp. 100-109, Feb. 1995.

[10] Z. Tang and G. Leus, "Receiver design for single-carrier transmission over time-varying channels," IEEE ICASSP 2007, Honolulu, Hawaii, Apr. 2007, vol. 3, pp. 129-132.

[11] B. Zheng, H. Zhang, and J. Sun, "Usability analysis of Ku wideband land model satellite communication system," IEEE PIMRC 2003, Beijing, China, Sept. 2003, pp. 693-696.

[12] A. Jahn, "Propagation considerations and fading countermeasures for mobile multimedia services," Int. Journal of Satellite Commun., vol. 19, pp. 223-250, May 2001.

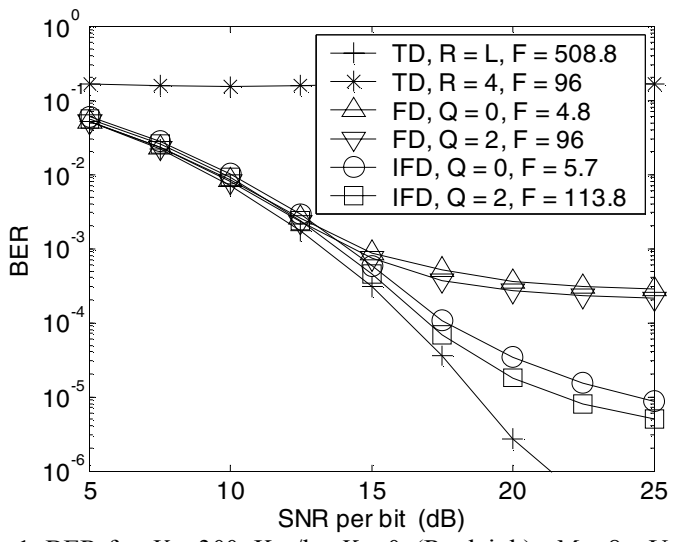

Fig. 1. BER for $V=300 \mathrm{Km} / \mathrm{h}, K=0$ (Rayleigh), $M=8, U=256$.

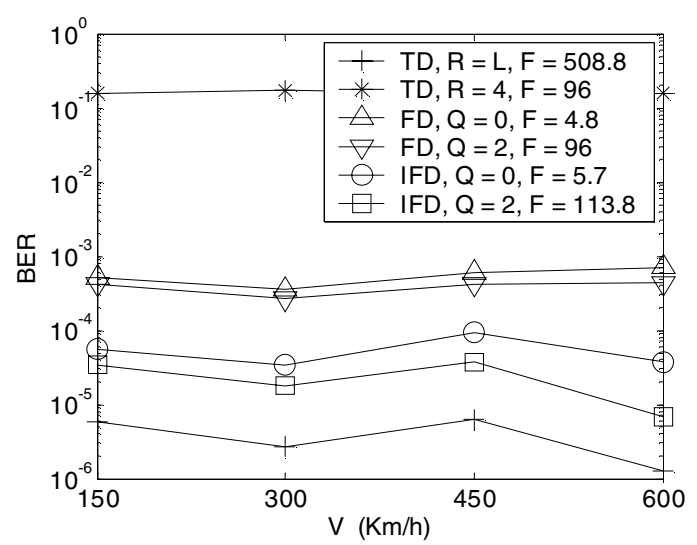

Fig. 2. BER for $\mathrm{SNR}=20 \mathrm{~dB}, K=0$ (Rayleigh), $M=8, U=256$.

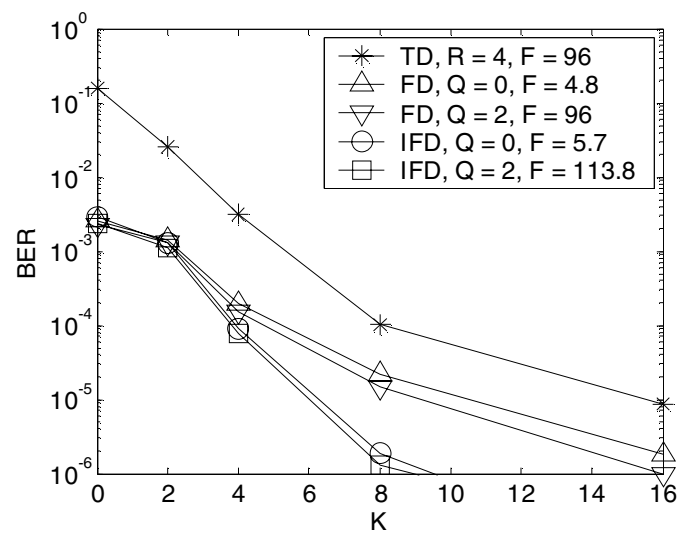

Fig. 3. BER for $V=300 \mathrm{Km} / \mathrm{h}, \mathrm{SNR}=12.5 \mathrm{~dB}, M=8, U=256$.

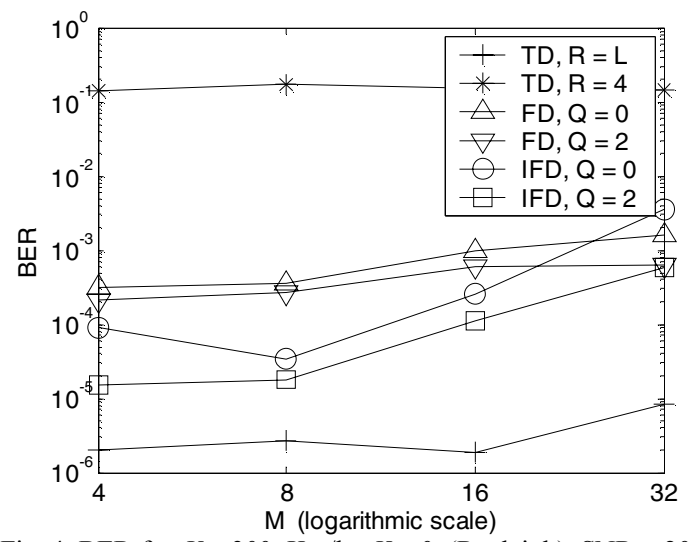

Fig. 4. BER for $V=300 \mathrm{Km} / \mathrm{h}, K=0$ (Rayleigh), $\mathrm{SNR}=20 \mathrm{~dB}$.

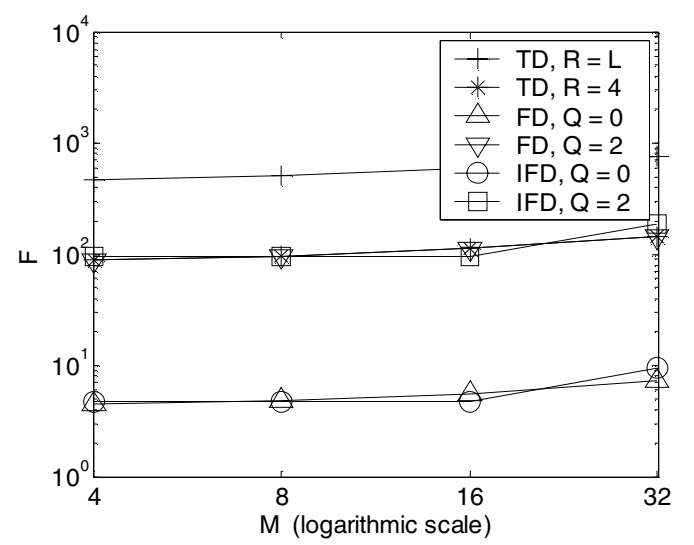

Fig. 5. Number of complex multiplications for the scenario of Fig. 4. 